\title{
Thermocapillary Actuation of Droplets on Chemically Patterned Surfaces by Programmable Microheater Arrays
}

\author{
Anton A. Darhuber, Joseph P. Valentino, Student Member, IEEE, Sandra M. Troian, and Sigurd Wagner, Fellow, IEEE
}

\begin{abstract}
We have designed a microfluidic device for the actuation of liquid droplets or continuous streams on a solid surface by means of integrated microheater arrays. The microheaters provide control of the surface temperature distribution with high spatial resolution. These temperature gradients locally alter the surface tension along droplets and thin films thus propelling the liquid toward the colder regions. In combination with liquophilic and liquophobic chemical surface patterning, this device can be used as a logistic platform for the parallel and automated routing, mixing and reacting of a multitude of liquid samples, including alkanes, poly(ethylene glycol) and water.

[1024]
\end{abstract}

Index Terms-Chemical surface patterning, free surface flows, microfluidics, thermocapillary actuation.

\section{INTRODUCTION}

A NUMBER of academic and industrial laboratories are specializing in the development and production of automated microfluidic technologies for analytical testing relevant to genomics, proteomics, environmental monitoring and biodefense. These devices rely on various actuation mechanisms for driving liquid streams or droplets through networked arrays. Mixing or separation protocols can simultaneously be applied for chemical reactions or species identification. Most propulsion mechanisms rely on internal flows [1]-[5] but some investigators are exploring advantages inherent in exterior flow using electrowetting [6], [7] and dielectrophoresis for droplet actuation [8]. An open flow architecture allows direct accessibility to analytes or reaction products for sample handling, optical measurements or diagnostics and requires few if any moving parts. While not suitable for highly volatile liquids, the continuous contact between the ambient gas and the microfluidic sample can be exploited for sensor applications. As long as there remains a free gas-liquid interface, the device can also be encapsulated to reduce evaporation and undesirable contamination.

Thermocapillary stresses caused by spatial variations of the surface tension $\gamma$ at a gas-liquid interface can induce spontaneous flow of a liquid film from warmer to cooler regions [9],

Manuscript received March 17, 2003; revised July 8, 2003. This work was supported by the National Science Foundation(CTS and DMR divisions), U.S. Army TACOM-ARDEC, and NASA (Fluid Physics division). Subject Editor N. de Rooij.

A. A. Darhuber and S. M. Troian are with the Microfluidic Research and Engineering Laboratory, School of Engineering and Applied Science, Princeton, NJ 08540 USA (webpage: www.princeton.edu/ stroian).

S. Wagner and J. P. Valentino are with the Department of Electrical Engineering, Princeton University, Princeton, NJ 08540 USA (e-mail: wagner@ princeton.edu).

Digital Object Identifier 10.1109/JMEMS.2003.820267
[10]. Essentially, a region of cooler surface temperature maintains a higher surface tension than neighboring warm spots. This tension exerts a tangential surface force that pulls liquid toward the cold spot. For uniformly flat and thin liquid films of height $h$ and viscosity $\mu$ subject to a thermal gradient $\nabla T$, the average flow speed is given by $v=h \tau / 2 \mu$ where the shear stress $\tau=|\nabla \gamma|=|d \gamma / d T| \cdot|\nabla T|$. This mechanism has been used to propel liquid films against the force of gravity [10], [11] and to move large droplets on homogeneous, partially wetting surfaces [12], (i.e., surfaces on which the static contact angle of the liquid droplet $\theta>0$ ). Liquid-solid pairs for which the static contact angle $\theta$ is identically zero are designated as completely wetting systems. In this manuscript the terminology liquophilic (liquophobic) designates a surface on which a liquid droplet maintains a low (high) static contact angle, i.e., the critical surface tension is close to (significantly below) the surface tension of the liquid [13]. The motion of liquid films can therefore be restricted to well defined trajectories by introducing chemically heterogeneous surfaces [14], [15]. Local control of surface temperature in combination with liquophilic/liquophobic surface patterning therefore provide an ideal system for microfluidic actuation, routing, mixing and reacting of minute quantities of liquid reagents with low operating voltages.

In this article, we describe the electronic hardware and software for controlling the surface temperature distribution of the fluidic device using arrays of resistive microheaters. Heat transfer simulations are used to optimize the heater design so as to prevent undesirable liquid trapping at local temperature minima. It is demonstrated that both completely and partially wetting liquids can be routed along a surface in fully automated fashion. Partially wetting surfaces are particulary attractive, as they allow transport without volume loss, thus virtually eliminating cross-contamination between individual droplets.

\section{DEVICE FABRICATION}

The device consists of arrays of metallic thin-film resistive heaters deposited on rigid or deformable substrates (e.g., Corning $1737 \mathrm{~F}$ glass slides of thickness $d_{\text {sub }}=0.7 \mathrm{~mm}$ ). The metal films are deposited with an electron beam evaporator (Model DV-502A, Denton Vacuum, NJ) and patterned by a photolithographic lift-off procedure. A layer of 15-95 nm Ti or Cr followed by 50-300 $\mathrm{nm}$ Au were deposited; the $\mathrm{Au}$ was etched away to define the microheaters by using TFA (Transene Company, Inc., MA). The electrical conductivity of $\mathrm{Au}\left(45.5 \times 10^{6} \Omega^{-1} \mathrm{~m}^{-1}\right.$ at $\left.300 \mathrm{~K}\right)$ is considerably higher than that of $\mathrm{Ti}\left(1.96 \times 10^{6} \Omega^{-1} \mathrm{~m}^{-1}\right.$ at $\left.300 \mathrm{~K}\right)$ and $\mathrm{Cr}$ 
$\left(7.63 \times 10^{6} \Omega^{-1} \mathrm{~m}^{-1}\right.$ at $\left.300 \mathrm{~K}\right)$ [16], which ensures that the contacts and leads contribute only minimally to the total resistance. The width of the resistors ranged from 100 to $1000 \mu \mathrm{m}$.

For electrical passivation, the metal resistors were covered by a layer of $200-800 \mathrm{~nm} \mathrm{SiO}_{2}$ using plasmaenhanced chemical vapor deposition (PECVD) in a Plasmatherm 790 (Unaxis Semiconductors, FL) at a temperature of $250{ }^{\circ} \mathrm{C}$. The $\mathrm{SiO}_{2}$ layer was deposited with gas flow rates of $160 \mathrm{sccm}$ for $\mathrm{N}_{2} \mathrm{O}$ and $35 \mathrm{sccm}$ for $\mathrm{SiH}_{4}$ at a chamber pressure of 400 mTorr and a power setting of $25 \mathrm{~W}$. Subsequently, the surface of the sample was lithographically patterned into liquophilic and liquophobic regions using a self-assembled monolayer of $1 \mathrm{H}, 1 \mathrm{H}, 2 \mathrm{H}, 2 \mathrm{H}$-perfluorooctyltrichlorosilane (PFOTS, Fluka) [17], [18]. Thus, the liquids do not spread onto the liquophobic PFOTS-coated regions but are instead confined to the liquophilic stripes. In these experiments, the stripes were located directly above the heating resistors. By varying the dc-voltage applied to the $i$ th resistor, the heating power $P_{i}$ and hence the local temperature $T_{i}(x)$ can be set with a time-constant entirely determined by the thickness and thermal properties of the substrate [19].

Fig. 1(a) is a schematic diagram of a liquid droplet moving along a liquophilic stripe parallel to the $\hat{x}$ direction. When the heater directly behind the droplet is activated, the liquid is propelled forward by the induced thermal gradient. Fig. 1(b) shows the layout of the contact leads and resistors of a device comprising two intersecting liquophilic stripes which connect four reservoir sites. In this example, the width of the resistive heater $w_{r}$ is $800 \mu \mathrm{m}$, the length $l_{r}$ is $3000 \mu \mathrm{m}$ and the gap $s$ between adjacent resistors ranges from $60-75 \mu \mathrm{m}$. A cross-sectional view of two embedded heaters beneath a liquid droplet is shown in Fig. 1(c). The overall device operating temperature $T_{0}$ is controlled by the heat sink (water-cooled metal block), which was equal to the ambient temperature $T_{\mathrm{amb}}$.

\section{Electronic CONTROL SETUP}

The electronic control setup is shown in Fig. 2(a). The left block designates a PC, which houses and addresses two 32-channel digital-to-analog (D/A) output boards (PD2-AO-32, United Electronic Industries) and a 96-channel digital input/output (DIO) board (PCI-DIO-96, National Instruments) via LabView 6.0 (National Instruments). The D/A channels are used as independent voltage sources for the individual heaters. The maximum output voltage and current of the D/A boards were $10 \mathrm{~V}$ and $90 \mathrm{~mA}$, respectively. These boards are connected to two identical printed circuit boards (PCBs) symbolized by the dashed block. The addressing scheme for a single contact of the device is shown in Fig. 2(b). The DIO board activates the output of 16 octal D-type flip-flops (SN74LS374, Motorola) on the PCB. The flip-flop outputs open or close independently addressable MOSFET switches (ADG452, Analog Devices), which are also mounted on the PCB. These switches can be activated in three different modes.

- When only switch (1) is activated, the contact is connected directly to the voltage output of the D/A board.

- When only switches (2) and (3) are activated, the resistive heater is in series with an ammeter (denoted by "A") and
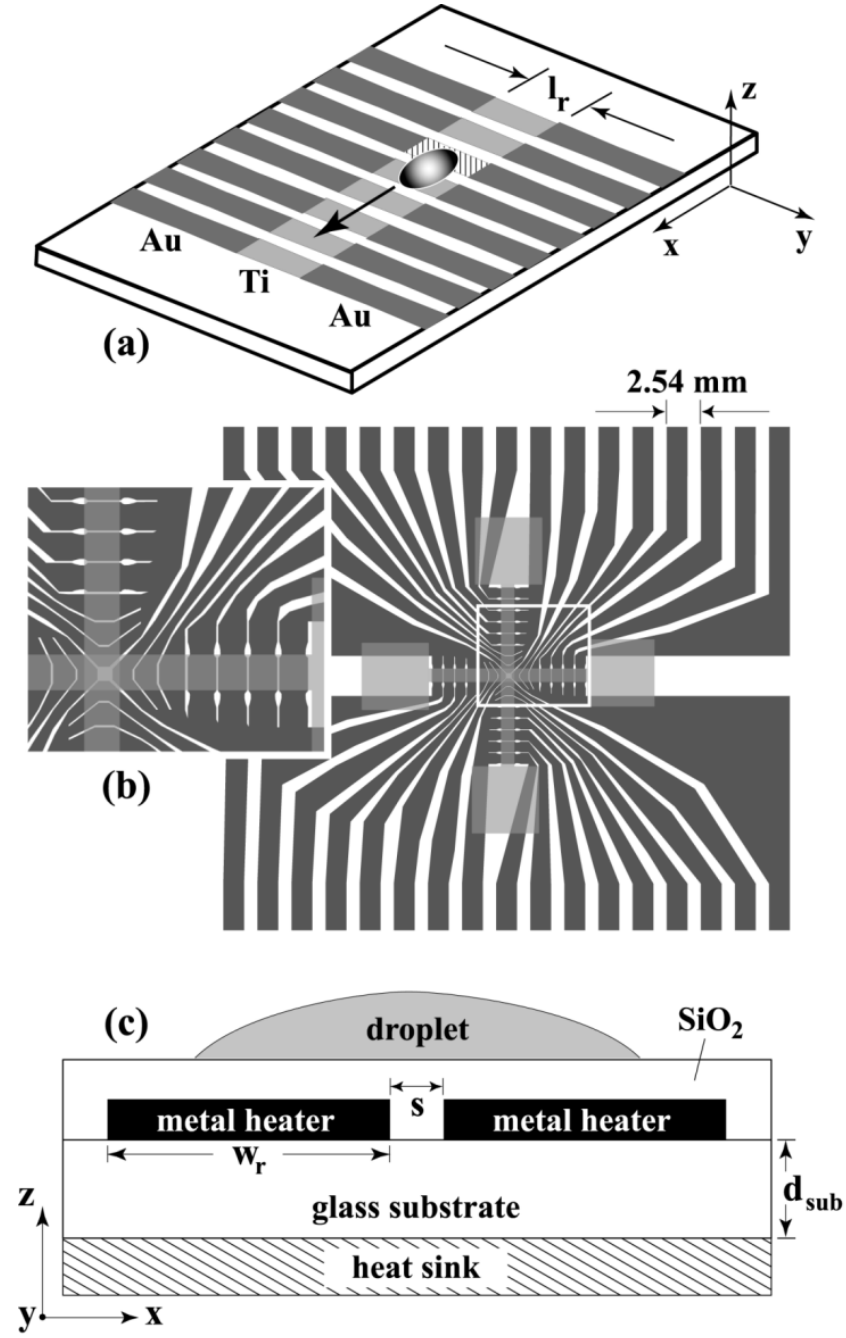

Fig. 1. (a) Top-view of a liquid droplet moving along a liquophilic microstripe. The array of Ti-resistors (shown in light gray) beneath the liquophilic stripes locally heat the droplet thereby modifying the surface tension and propelling the liquid toward the colder regions of the device surface. The dark gray stripes represent the leads and contacts $(\mathrm{Au})$ for the heating resistors. (b) Top-view of the resistor and contact layout for a proof-of-concept device with two intersecting liquophilic channels connecting four liquophilic reservoir pads (shown in semitransparent light gray). The central region inside the white rectangle is magnified in the inset. (c) Cross-sectional view of a portion of the device containing two microheaters and an overlying droplet. The thickness of the glass substrate $d_{\text {sub }}$ is typically $0.7 \mathrm{~mm}$. The thickness of the metal heaters and the silicon oxide passivation layers ranged from $40-100 \mathrm{~nm}$ and $200-600 \mathrm{~nm}$, respectively.

the D/A output line. This circuit provides feedback control by monitoring the change in resistance of the heating elements due to a change in temperature.

- When only switch (4) is activated, the contact to the heater is shorted to ground.

The configuration shown in Fig. 2(b) is applied identically to the second PCB on the right side of the device. Each PCB is connected to the device via a 50-pin card edge connector with $2.54 \mathrm{~mm}$ (0.1 inch) contact spacing, into which the glass sample is inserted. The connectors limit the substrate thickness $d_{\text {sub }}$ to below $0.8 \mathrm{~mm}$ to avoid excessive abrasion of the gold contacts during sample insertion or removal.

The maximum number of addressable resistors is $N-1$, where $N$ is the number of gold contacts on the sample. The 


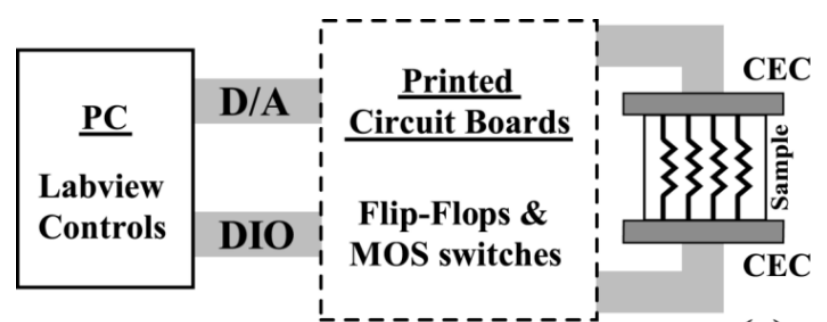

(a)
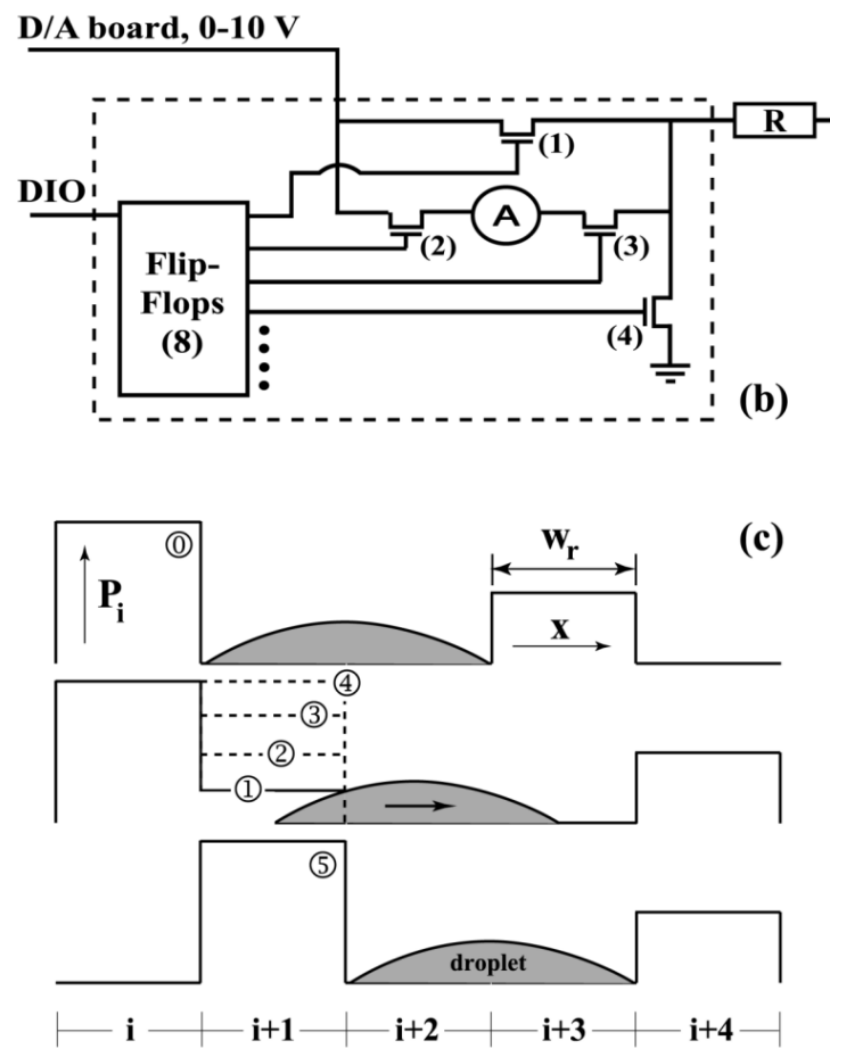

Fig. 2. Schematic diagrams of the electronic control setup for the microheater arrays. (a) The block diagram shows flip-flops and MOS switches on two custom-made printed circuit boards (PCB's) which were controlled by LabView 6.0 via a 96-channel digital input/output board (DIO). The ouput of the two 32-channel digital-to-analog (D/A) output boards was routed through the switching network to the device which was connected to the PCB's by means of two 50-pin card edge connectors (CEC). (b) Layout of a circuit element for addressing one contact. An octal flip-flop is used to control switches (1) to (4) which either set the contact to ground or connect it to the output of the D/A board. Activation of switches (2) and (3) routes the electrical current through an ammeter (labeled A) for feedback control. (c) Five step addressing sequence for moving a droplet a distance $\Delta x=w_{r}$ (one pixel). At $t=t_{0}$ the $i$ th resistor which is located behind the droplet is activated with a power $P_{i}=P_{\max }$. At subsequent times $t=t_{0}+n \Delta t$, where $n=1-4$ (encircled numbers), $P_{i+1}$ is increased in step-wise fashion from 0 to $P_{\max }$.

subtraction ' -1 ' accounts for a single shared ground contact. Since with one level of metallization, it is difficult to connect all resistors to a single ground contact, we instead used one or more ground contacts on either side of the sample. To enable control of arbitrary heater layouts, the electronic circuit for selecting and powering the resistors was identical for all contacts and symmetric on both sides of the device.

Fig. 2(c) shows a typical addressing sequence for moving a droplet along a liquophilic stripe. At $t=t_{0}$ a single resistor behind the droplet is activated with a power $P_{i}=P_{\max }$ where the index $i$ refers to the $i$ th heater. The resistor ahead of the droplet is powered at a lower value to prevent uncontrolled spreading such that the droplet position is known with a precision $\Delta x \approx w_{r}$ at any time. This scheme is advantageous for the simultaneous routing of multitudes of droplets. For subsequent times $t=t_{0}+n \Delta t$, where $n=1-4$ and $\Delta t$ typically ranges from 1 to $20 \mathrm{~s}, P_{i+1}$ is increased in step-wise fashion from 0 to $P_{\max }$. At the same time, the thermal barrier at the front of the droplet is shifted forward by one resistor. When $P_{i+1}$ has reached a value of $P_{\max }$, the $i$ th heater is switched off, after which the droplet has migrated a distance $\Delta x=w_{r}$. This activation cycle is then repeated with the next set of resistors. Numerical simulations indicate that the thermal equilibration time after resistor activation for $d_{\text {sub }}=0.7 \mathrm{~mm}$ and $w_{r}=800 \mu \mathrm{m}$ [19] is about $0.2 \mathrm{~s}$. The temperature rise and decay can therefore be considered instantaneous.

For migration by thermocapillary stresses, the speed of a droplet is inversely proportional to the viscosity and linearly proportional to the product $(d \gamma / d T) \cdot(d T / d x)$ and the droplet height [9]. The local gradient $d T / d x$ is proportional to $P_{\max }$ illustrating the tradeoff between low power consumption and high streaming velocity.

\section{Heat Transfer Simulations}

Finite element calculations of surface temperature distributions were conducted to identify the optimal heater design and operational modes. In our experiments, both the dimensionless Peclet and Biot numbers $\left(\mathrm{Pe}=U h / \alpha\right.$ and $\mathrm{Bi}=h_{\text {conv }} h / k_{\text {liquid }}$ where $U$ is the migration speed, $\alpha$ the liquid thermal diffusivity, $h_{\text {conv }}$ the convective heat transfer coefficient of air and $k_{\text {liquid }}$ the liquid thermal conductivity) are small. Therefore the droplet surface temperature is essentially equal to the local temperature of the substrate surface. All convective effects induced by liquid motion can therefore be neglected to first order and only the effects of heat conduction need be considered.

Local minima in the temperature profile $T(x)$ must be avoided as they can trap liquid in undesired locations. The three factors controlling the monotonicity of thermal distributions are the spacing between heaters, $s$, the thickness of the substrate $d_{\text {sub }}$ (which determines the achievable spatial resolution), and the ratio of electrical power supplied to two neighboring heaters $P_{i} / P_{i+1}$. Fig. 3(a) depicts steady-state 2-D simulations of the surface temperature for the case of two adjacent resistors with spacing $s=60 \mu \mathrm{m}$ activated with a power ratio ranging from 1:0 to 1:1. Fig. 3(b) represents a magnified view of the ratio 1:0.7 in which the heater spacing varies from 20-100 $\mu \mathrm{m}$. For large spacing $s$ and a power ratio $P_{i} / P_{i+1}$ close to one, local minima develop in $T(x)$. The gap width $s$ is only limited by the lithographic resolution, which in our case is of the order of $40 \mu \mathrm{m}$ due to the imagesetter used for printing the photomasks. For the set of parameters used in Fig. 3(a) and (b), $P_{i} / P_{i+1}$ must be larger than about 2.4 (or smaller than 0.45 ) to ensure a monotonic variation in the surface temperature.

The heat transfer simulations in Fig. 3(a) and (b) assume a 2-D geometry, i.e., $l_{r} \gg d_{\text {sub }}$ [see Fig. 1(a)]. In practice, this restriction requires $l_{r} \geq 6 \mathrm{~mm}$ for a substrate thickness of $0.7 \mathrm{~mm}$ [19]. To minimize the footprint of the heater array, it is desirable to reduce $l_{r}$ as much as possible, the lower limit being 

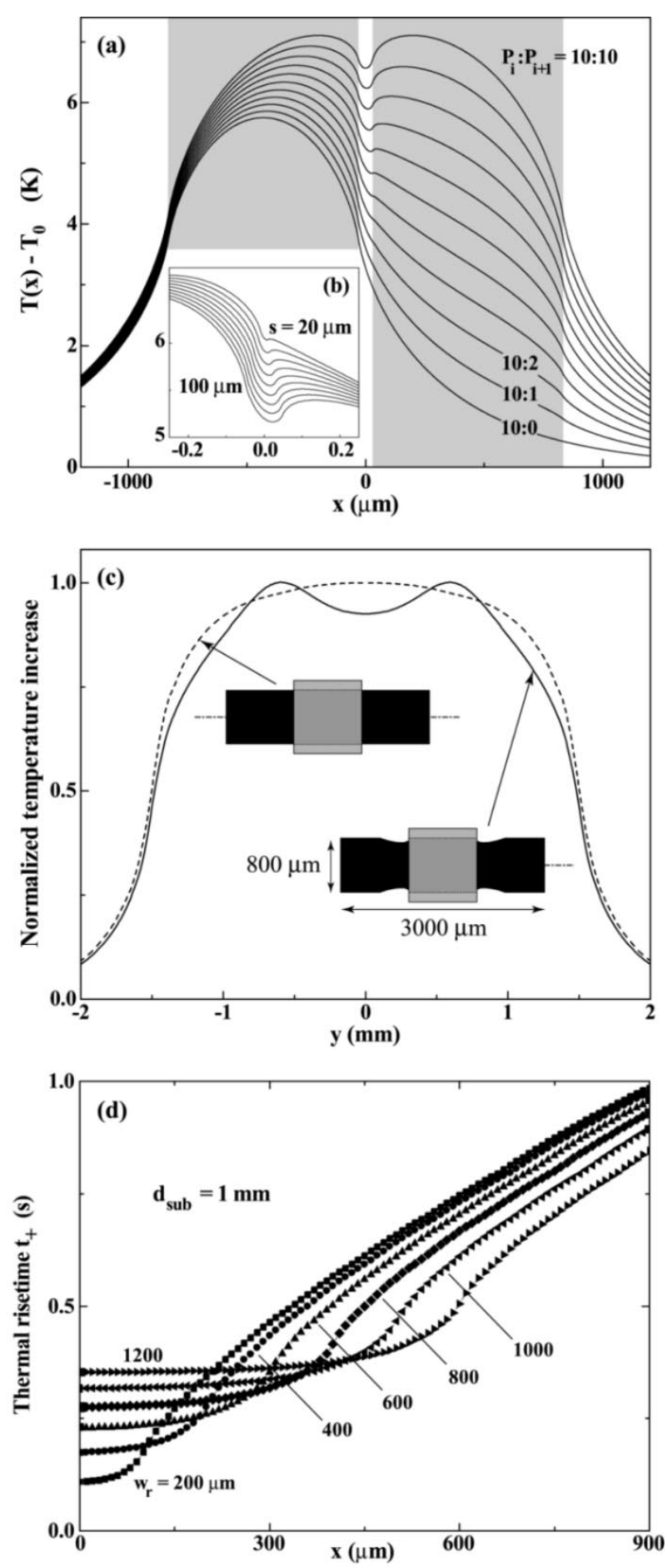

Fig. 3. Finite element simulations of the surface temperature. (a) $\Delta T=$ $T\left(x, y=0, z=d_{\mathrm{sub}}\right)-T_{0}$ as a function of the ratio of heating power of adjacent resistors $P_{i} / P_{i+1}$ for $w_{r}=800 \mu \mathrm{m}, s=60 \mu \mathrm{m}, d_{\text {sub }}=0.7 \mathrm{~mm}$ and $P_{i} / l_{r}=100 \mathrm{~mW} / \mathrm{cm}$. For $P_{i} / P_{i+1} \geq 0.5$, local temperature minima develop, which can trap liquid between resistors. The gray-shaded areas denote the extension of the heating resistors. (b) Influence of the gapwidth $s$ between neighboring heaters for $P_{i} / P_{i+1}=0.7$. The gap widths range from 20 to $100 \mu \mathrm{m}$ in steps of $10 \mu \mathrm{m}$ and should be minimized to avoid undesirable local temperature minima. (c) Normalized temperature increase $\left[T\left(x=0, y, z=d_{\mathrm{sub}}\right)-T_{0}\right] /\left(T_{\max }-T_{0}\right)$ with (full line) and without (dashed line) $65 \mu \mathrm{m}$ deep and $450 \mu \mathrm{m}$ long constrictions for $w_{r}=800 \mu \mathrm{m}$, $l_{r}=3000 \mu \mathrm{m}$ and $d_{\text {sub }}=0.7 \mathrm{~mm}$. The semitransparent light-gray rectangles represent the liquophilic stripes. The horizontal dash-dotted lines indicate the locations where the temperature profiles are extracted. (d) Thermal risetime $t_{+}$ as a function of $x$ for $d_{\text {sub }}=1 \mathrm{~mm}$ and $w_{r}$ ranging from 200 to $1200 \mu \mathrm{m}$. The position $x=0$ corresponds to the center of the heaters. the liquophilic stripe width $w_{c}$. Shorter heater lengths, however, lead to a nonuniform temperature distribution in the $y$-direction, as shown by the dashed line in Fig. 3(c). The induced outward temperature gradients $d T / d y$ will cause undesirable accumulation of liquid at the stripe edges.

To reduce $l_{r}$ while avoiding transverse gradients, local constrictions in the heater width were introduced close to the edges of the liquophilic stripes. This local narrowing increased the Joule dissipation density at the stripe edges, creating temperature maxima near the stripe boundaries. Fig. 3(c) shows the normalized thermal profiles, $\left[T(y)-T_{0}\right] /\left(T_{\max }-T_{0}\right)$, along the resistor midline with and without constrictions. Here, $T_{\max }$ denotes the maximum value of $T(y)$. In these 2-D simulations, the steady-state current density $\mathbf{j}=\sigma \mathbf{E}$ was first determined according to

$$
0=\nabla \cdot \mathbf{j}=\nabla \cdot(\sigma \mathbf{E})=-\nabla \cdot(\sigma \nabla V)
$$

where $\sigma$ is the electrical conductivity, $\mathbf{E}=-\nabla V$ is the electric field, and $V$ the electrical potential. The resistive energy dissipation density

$$
\dot{q}=\frac{1}{\sigma} j^{2}=\frac{|\sigma \mathbf{E}|^{2}}{\sigma}=\sigma|\nabla V|^{2}
$$

was used to solve the 3-D steady-state heat conduction equation

$$
\nabla \cdot(k \nabla T)+\dot{q}=0,
$$

where $k=1.0 \mathrm{~W} / \mathrm{mK}$ is the thermal conductivity of the glass substrate. The electrical conductivity was assumed to be temperature independent. The results shown in Fig. 3(c) indicate that the heater constrictions lead to a local minimum which helps center the droplet on the liquophilic stripe. This minimum also generates a transverse convective flow which enhances the mixing of two liquids in a droplet [20].

A rapid thermal response is an important consideration in the design of the microheaters. In Fig. 3(d) is shown the thermal risetime $t_{+}$corresponding to a step change in the electrical current through a single resistor. The risetime $t_{+}$is defined as the interval over which the local surface temperature $T(x, y, z=$ $\left.d_{\mathrm{sub}}, t\right)$ changes by $63.21 \%$ of $T\left(x, y, d_{\mathrm{sub}}, t \rightarrow \infty\right)-T_{0}$. As discussed in [19], the temperature profile $T(x)$ has a maximum in the center of the resistor at $x=0$ and decays over a lateral lengthscale $\xi_{l} \approx d_{\text {sub }}$ for $x>w_{r} / 2$. Consequently, $t_{+}(x)$ has a minimum at $x=0$, since there is no lateral heat flux at the center point. The largest temperature gradients occur at $x=w_{r} / 2$, the most relevant location for thermocapillary transport. Here the risetime $t_{+}\left(x=w_{r} / 2\right)$ can be significantly higher than in the center of the heater. Although $t_{+}(x)$ keeps increasing for $x>w_{r} / 2$, the effective risetime is always smaller than $1 \mathrm{~s}$ for all simulations shown in Fig. 3(d), since both $T(x)$ and $d T / d x$ decay rather rapidly beyond $x>w_{r} / 2$.

\section{EXPERIMENTAL RESULTS}

\section{A. Completely Wetting Liquids}

Fig. 4(a)-(d) shows the migration of a droplet of polydimethylsiloxane (PDMS) on a $1000 \mu \mathrm{m}$ wide liquophilic 


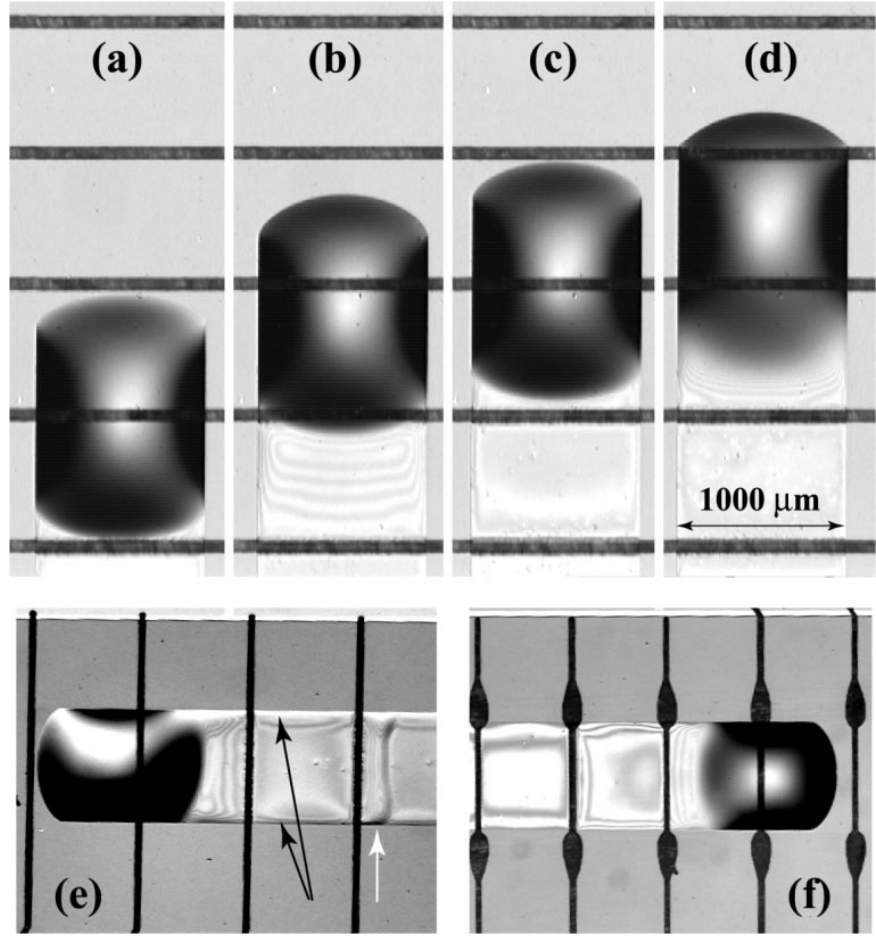

Fig. 4. (a)-(d) Droplet of PDMS moving along a heater array with $w_{r}=$ $700 \mu \mathrm{m}, l_{r}=9 \mathrm{~mm}, s=80 \mu \mathrm{m}, \Delta t=22 \mathrm{~s}, V_{i}=5.7 \mathrm{~V}$ and heater resistance $R=1.2 \mathrm{k} \Omega$. A thin residue trailed the wetting droplet. The images sequence represents times (a) $t=0 s$, (b) $44 \mathrm{~s}$, (c) $88 \mathrm{~s}$, and (d) $132 \mathrm{~s}$. (e) Liquid accumulated at the stripe edges (black arrows) for $l_{r}=2800 \mu \mathrm{m}$ due to the transverse thermal gradient. The white arrows indicate a ridge of liquid that had previously been trapped in the local temperature minimum between the heaters which was later driven away when the right resistor was deactivated. (f) No accumulation of liquid at the stripe edges was observed for $l_{r}=3000 \mu \mathrm{m}$ after introducing local constrictions in the heater width. The width of the liquophilic stripes, to which the liquid is confined, is $1000 \mu \mathrm{m}$ for all images shown.

TABLE I

VISCOSITY $\mu$, SURFACE TENSION $\gamma$, AND VAPOR PRESSURE $p_{\text {vap }}$ OF THE LIQUIDS USED FOR THERMOCAPILLARY ACTUATION. THE UNITS FOR $\gamma$ AND $\partial \gamma / \partial T$ ARE $m \mathrm{~N} / \mathrm{m}$ AND $m \mathrm{~N} / m \mathrm{~K}$, RESPECTIVELY. THE DATA ARE TAKEN FROM [25] AND [26] AND ARE VALID FOR A TEMPERATURE OF $25^{\circ} \mathrm{C}$

\begin{tabular}{lcccc}
\hline \hline Liquid & $\mu(m \mathrm{Pas})$ & $\gamma$ & $\partial \gamma / \partial T$ & $p_{\text {vap }}(\mathrm{Pa})$ \\
\hline PDMS & 20.0 & 20.2 & -0.060 & $<1$ \\
Dodecane & 1.38 & 25.3 & -0.0894 & $<11$ \\
DEG & 30.2 & 44.77 & -0.0889 & $<0.7$ \\
Water & 0.89 & 72.0 & -0.162 & 3170 \\
\hline \hline
\end{tabular}

stripe located above a microheater array with $w_{r}=700 \mu \mathrm{m}$ and $l_{r}=9 \mathrm{~mm}$. The material properties of the liquids used in this study are summarized in Table I. The time interval $\Delta t$ was $22 \mathrm{~s}$ and the voltage $5.7 \mathrm{~V}$ corresponding to a power dissipation $P_{i}$ of $27 \mathrm{~mW}$. A thin residual film trailed the droplet because PDMS completely wets the liquophilic stripe surface. This residue, which would induce cross-contamination between subsequent droplets, must be minimized or eliminated altogether. The residue can be reduced by increasing $\Delta t$ or $P_{i}$. We found that a droplet of dodecane following a PDMS droplet eliminated the PDMS residue very effectively, such that no remaining traces were visible with an optical microscope.

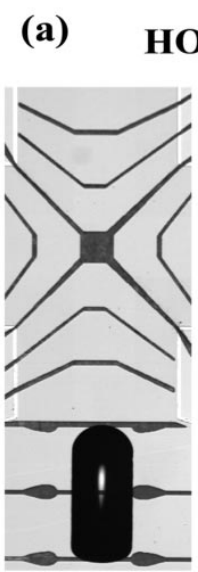

(b)

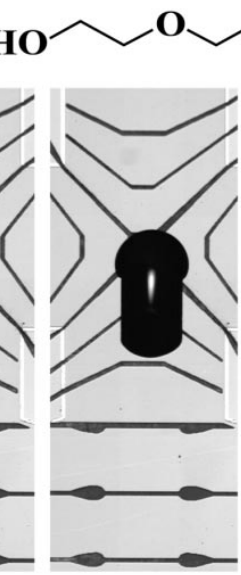

(c)

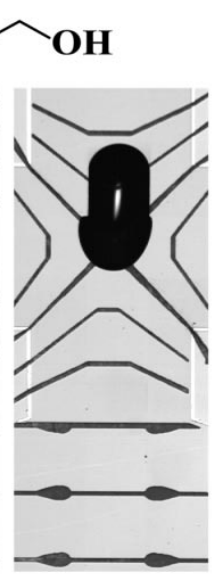

(d)

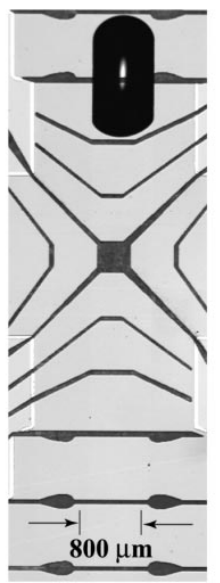

(e)
Fig. 5. (a) Molecular structure of di(ethylene glycol) (DEG). (b)-(e) A droplet of DEG moving straight through the intersection of two liquophilic stripes. The driving voltage was $6 \mathrm{~V}$ and the heater resistance approximately $150 \Omega$. The images were recorded at (b) $t=0 \mathrm{~s}$, (c) $140 \mathrm{~s}$, (d) $230 \mathrm{~s}$, and (e) $330 \mathrm{~s}$.

If the heater length $l_{r}$ is reduced to $2.8 \mathrm{~mm}$, an accumulation of liquid at the edges of the liquophilic stripe occurred as indicated by the black arrows in Fig. 4(e). The white arrow in Fig. 4(e) marks a small ridge of liquid which had previously been trapped in the local temperature minimum in the gap between two resistors. When the resistor to the left of it was powered, the ridge was driven toward the right. As discussed in Section IV, the introduction of heater indentations close to the edges of the liquophilic stripes reverses the direction of the transverse temperature gradients and eliminates this accumulation at the boundaries, as shown in Fig. 4(f).

\section{B. Partially Wetting Liquids}

As shown in Fig. 4 completely wetting liquids leave behind a residue because their receding contact angle is zero. Since partially wetting liquids maintain a finite contact angle, droplets can migrate without volume loss, thus virtually eliminating cross-contamination. To increase the (static) contact angle of dodecane on the liquophilic microstripe, we rinsed the device surface with hexamethyl disiloxane and dried in a filtered nitrogen stream. Since the receding angle of a moving droplet is known to decrease with increasing speed, migration without loss of liquid can only occur for sufficiently small droplet speeds. Droplets of dodecane were actuated with a voltage of only $2.5 \mathrm{~V}$ for a heater resistance of $150 \Omega$ [20]. Such low operating voltages make battery-powered operation of the device feasible.

\section{Biochemically Relevant Liquids}

Both PDMS and dodecane are nonpolar and immiscible with water, which restricts their suitability for biochemical applications. Poly(ethylene glycol) is miscible with water in all proportions. This liquid is commonly used as cell cryoprotectant by preventing extracellular ice crystallization [21], [22]. The solubility of salts in ethylene glycol (EG) is appreciable: 20.6 $\mathrm{g} \mathrm{CaCl}_{2}$ and $7.5 \mathrm{~g} \mathrm{NaCl}$ dissolve in $100 \mathrm{~g} \mathrm{EG}$ at $25^{\circ} \mathrm{C}$ [23]. 


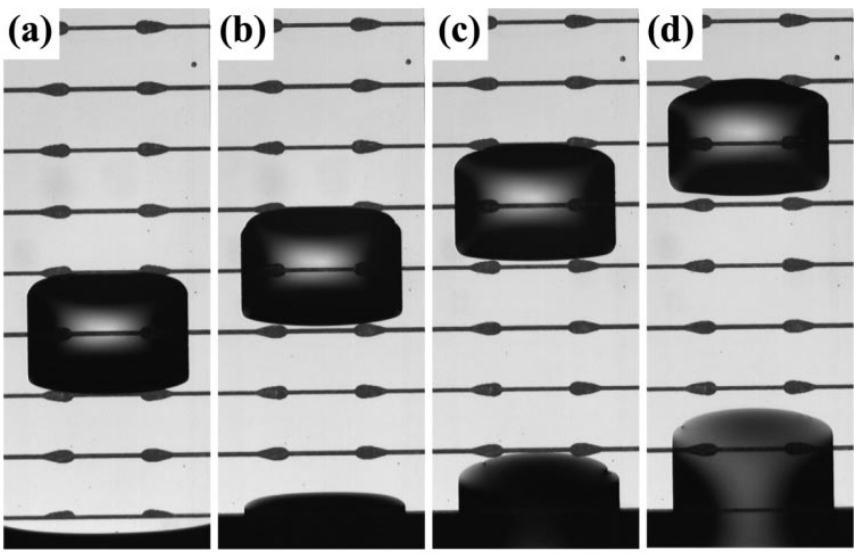

Fig. 6. (a)-(d) A droplet of water moving on a 2-mm-wide microstripe. The driving voltage was $6 \mathrm{~V}$ and the time between subsequent images was $3 \mathrm{~s}$.

Poly(ethylene glycol) grafted onto solid surfaces is also used to reduce protein adsorption [24].

To increase the (static) contact angle of di(ethylene glycol) (DEG) on the liquophilic microstripe, the device surface was rinsed with heptane and dried as before. Fig. 5 shows a droplet of DEG moving straight through the intersection of two liquophilic stripes. The design and arrangement of the metal heaters in the intersection must provide heat sources which are rather close to the center point since it might otherwise not be possible to remove liquid from the junction region once it has entered the intersection. With a single level of metallization, this requires the heater shape to be bowed and the resistor width $w_{r}$ to be higher in the center of the liquophilic stripes [see inset in Fig. 1(b)]. This heater geometry induces an asymmetric temperature profile, which directs the liquid into the junction region. In order to withdraw the liquid again, the separation of the innermost resistors must be smaller than the spatial resolution (decay length) of the temperature distribution, which is essentially equal to the substrate thickness [19].

As in the case of dodecane, no residual film was visible behind the DEG droplets. However, the voltage required to actuate a droplet of comparable size was about $6 \mathrm{~V}$ (cf. $2.5 \mathrm{~V}$ for dodecane) despite the fact that $d \gamma / d T$ is essentially identical for both liquids $(-0.0889 \mathrm{mN} / \mathrm{mK}$ for DEG and $-0.0894 \mathrm{mN} / \mathrm{mK}$ for dodecane). We attribute this five to six-fold increase in the minimum required temperature gradient to higher contact angle hysteresis caused by enhanced molecular interactions of the polar alcohol and ether groups in DEG with nanoscopic chemical heterogeneities on the stripe surface. Such hysteretic effects are especially prominent with water [5], the liquid most relevant to biological and biochemical reactions.

In Fig. 6, the heater array near the reservoir was activated in such a way as to draw a water rivulet onto a 2 -mm-wide microstripe, to split a droplet from the meniscus (shown at the bottom of each panel) and to propel it along the microstripe. The time delay between subsequent images is $3 \mathrm{~s}$ with a driving voltage of $6 \mathrm{~V}$. The vapor pressure of water at ambient pressure is rather high compared to the liquids described previously, which might be problematic in applications. There are, however, two strategies for reducing significant evaporative losses during transport. Since the propulsion mechanism is linearly proportional to the overall thermal gradient across the droplet surface, the overall device temperature can be dropped to a value close to the melting point. The vapor pressure of water reduced by a factor of five in going from $25^{\circ} \mathrm{C}$ to $1{ }^{\circ} \mathrm{C}$ [1]. In addition, the entire device can be encapsulated and the interior atmosphere humidified with water vapor. This may induce condensation of water near the cold regions of the substrate. If liquid droplets contain solutes of low vapor pressure, then such water evaporation/condensation will not induce cross-contamination.

\section{SUMMARY}

We have developed a novel microfluidic device based on thermocapillary flow which can route liquid droplets and films through lithographically defined networks of liquophilic microstripes. The electronic configuration allows independent control of individual microheaters with a rapid response time. Improvements in microheater design and operational modes were obtained by finite element simulations of surface temperature distributions. The open architecture of this device facilitates dispensing and liquid handling and provides easy accessibility to the reaction products. The low operating voltages enable battery-powered chemical analysis or synthesis. Future studies will focus on minimizing power consumption, introducing multiple levels of metallization and an active-matrix addressing scheme, and integration of an automated dispensing system.

\section{REFERENCES}

[1] J. M. Ramsey, S. C. Jacobson, and M. R. Knapp, "Microfabricated chemical measurement systems," Nature Med., vol. 1, pp. 1093-1096, 1995.

[2] G. H. W. Sanders and A. Manz, "Chip-based microsystems for genomic and proteomic analysis," Trends Anal. Chem., vol. 19, pp. 364-378, 2000.

[3] W. Ritchie, "Experimental research in voltaic electricity and electromagnetism," Philos. Trans. R. Soc. London, vol. 122, pp. 279-298, 1832.

[4] J. Jang and S. S. Lee, "Theoretical and experimental study of MHD magnetohydrodynamic micropump," Sens. Actuators A, Phys., vol. 80, pp. $84-89,2000$

[5] T. S. Sammarco and M. A. Burns, "Thermocapillary pumping of discrete drops in microfabricated analysis devices," AIChE J., vol. 45, pp. 350-366, 1999 .

[6] J. Lee and C.-J. Kim, "Surface-tension-driven microactuation based on continuous electrowetting," J. Microelectromech. Syst., vol. 9, pp. 171-180, 2000.

[7] M. G. Pollack, R. B. Fair, and A. D. Shenderov, "Electrowetting-based actuation of liquid droplets for microfluidic applications," Appl. Phys. Lett., vol. 77, pp. 1725-1726, 2000.

[8] T. B. Jones, M. Gunji, M. Washizu, and M. J. Feldman, "Dielectrophoretic liquid actuation and nanodroplet formation," J. Appl. Phys., vol. 89, pp. 1441-1448, 2001.

[9] V. G. Levich, Physicochemical Hydrodynamics. Englewood Cliffs, NJ: Prentice Hall, 1962.

[10] V. Ludviksson and E. N. Lightfoot, "The dynamics of thin liquid films in the presence of surface-tension gradients," AIChE J., vol. 17, pp. 1166-1173, 1971.

[11] A.-M. Cazabat, F. Heslot, S. M. Troian, and P. Carles, "Fingering instability of thin spreading films driven by temperature gradients," Nature, vol. 346, pp. 824-826, 1990.

[12] J. B. Brzoska, F. Brochard-Wyart, and F. Rondelez, "Motions of droplets on hydrophobic model surfaces induced by thermal gradients," Langmuir, vol. 9, pp. 2220-2224, 1993.

[13] A. W. Adamson, Physical Chemistry of Surfaces. New York: Wiley, 1990.

[14] D. E. Kataoka and S. M. Troian, "Patterning liquid flow on the microscopic scale," Nature, vol. 402, pp. 794-797, 1999. 
[15] A. A. Darhuber, S. M. Troian, and W. W. Reisner, "Dynamics of capillary spreading along hydrophilic microstripes," Phys. Rev. E, vol. 64 pp. 1603-1610, 2001.

[16] G. T. Dyos and T. Farrell, Eds., Electrical Resistivity Handbook. New York: Peregrinus, 1992.

[17] U. Srinivasan, M. R. Houston, R. T. Howe, and R. Maboudian, "Alkyltrichlorosilane-based self-assembled monolayer films for stiction reduction in silicon micromachines," J. Microelectromech. Syst., vol. 7, pp. 252-260, 1998.

[18] B. C. Bunker, R. W. Carpick, R. A. Assink, M. L. Thomas, M. G. Hankins, J. A. Voigt, D. Sipola, M. P. de Boer, and G. L. Gulley, "The impact of solution agglomeration on the deposition of self-assembled monolayers," Langmuir, vol. 16, pp. 7742-7751, 2000.

[19] A. A. Darhuber, S. M. Troian, and S. Wagner, "Generation of high-resolution surface temperature distributions," J. Appl. Phys., vol. 91, pp. $5686-5693,2002$

[20] A. A. Darhuber, J. P. Valentino, J. M. Davis, S. M. Troian, and S. Wagner, "Microfluidic actuation by modulation of surface stresses," Appl. Phys. Lett., vol. 82, pp. 657-659, 2003.

[21] V. L. Bronstein, Y. A. Itkin, and G. S. Ishkov, "Rejection and capture of cells by ice crystals on freezing aqueous solutions," J. Cryst. Growth, vol. 52, pp. 345-349, 1981.

[22] P. Boutron, "Glass-forming tendency, stability of the amorphous state, and cryoprotection of red blood cells," J. Phys. Chem., vol. 87, pp. 4273-4276, 1983

[23] W. F. Linke and A. Seidell, Eds., Solubilities-Inorganic and MetalOrganic Compounds. Washington, DC: American Chemical Society, 1958

[24] X.-Y. Zhu, Y. Jun, D. R. Staarup, R. C. Major, S. Danielson, V. Boiadjiev, W. L. Gladfelter, B. C. Bunker, and A. Guo, "Grafting of high-density poly(ethylene glycol) monolayers on $\mathrm{Si}(111)$," Langmuir, vol. 17, pp. 7798-7803, 2001.

[25] CRC Handbook of Chemistry and Physics, D. R. Lide, Ed., CRC, Boca Raton, FL, 2002.

[26] J. J. Jasper, "The surface tension of pure liquid compounds," J. Phys. Chem. Ref. Data, vol. 1, pp. 841-1009, 1972.

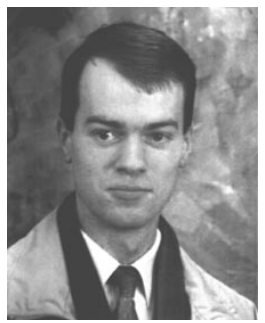

Anton A. Darhuber received the Ph.D. degree in engineering physics from the University of Linz, Austria, in 1998.

In the following year, he received an Erwin Schrödinger Fellowship from the Austrian FWF and joined the Microfluidic Research \& Engineering Laboratory at Princeton University, Princeton, NJ, as a Postdoctoral Fellow. In 2001, he became a Research Staff Member in the School of Engineering and Applied Sciences. He has published more than 40 technical papers and his research interests focus on the dynamics of liquids on micropatterned surfaces.

Dr. Darhuber received the Solid State Physics Award of the Austrian Physical Society in 1998.

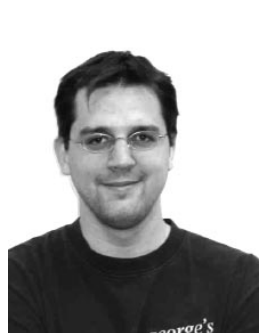

Joseph P. Valentino ( $\mathrm{S}^{\prime} 02$ ) received the B.S. degree in electrical engineering from Villanova University, Villanova, PA, in May 2000. He is currently pursuing the Ph.D. degree in electrical engineering at Princeton University, Princeton, NJ, in the Microfluidics Research and Engineering Laboratory.

His research interests are in the area of MEMS with a focus on microfluidics.

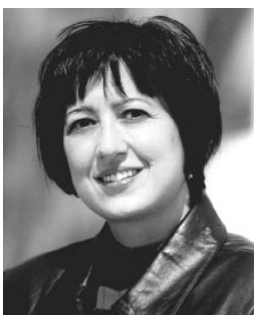

Sandra M. Troian received the Ph.D. degree in physics from Cornell University, Ithaca, NY, in 1987.

She then served as Postdoctoral Research Associate at the Corporate Research Labs of the Exxon Research and Engineering Company from 1987 to 1989 and as a Chateaubriand Fellow at the Collège de France in Paris from 1989 to 1990. She returned to Exxon Corporate Research as a Staff Physicist in the Polymers and Fluids Group in 1991 and joined the faculty at Princeton University, Princeton, NJ, in 1993. She is currently Professor of Chemical Engineering and associated faculty member in the Department of Physics and the Program in Applied and Computational Mathematics. She directs the Princeton Microfluidic Research \& Engineering Laboratory whose focus is the study of transport phenomena in microand nanofluidic systems. Her current projects include the fabrication of a microfluidic chip using patterned thermal fields, development of contact printing techniques for fabricating microelectronic devices, and characterization of slip behavior in submicron liquid films.

Dr. Troian was recipient of the 1999 Francois Frenkiel Award from the American Physical Society for her work on free surface flows.

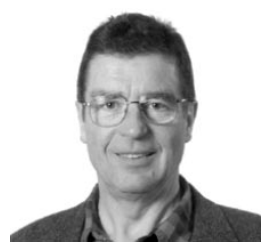

Sigurd Wagner (SM'78-F'00) received the Ph.D. degree from the University of Vienna, Austria, in 1968

He is helping to lay groundwork for the new industry of macroelectronics by developing concepts, materials, devices, and processes for large-area electronics. He worked from 1970 to 1978 at the Bell Telephone Laboratories on semiconductor memories and heterojunction solar cells. From 1978 to 1980, he was Chief of the Photovoltaic Research Branch, Solar Energy Research Institute, Golden, CO. Since 1980, he has been Professor of Electrical Engineering at Princeton University, Princeton, NJ, where he conducts a program on thin-film electronics with current emphasis on flexible electronics, on novel processes for silicon thin film transistors on plastic and steel, and on the direct printing of device materials. He holds 12 U.S. patents and has published about 400 technical papers.

Dr. Wagner is a Fellow of the American Physical Society and the Humboldt Foundation, and is a Corresponding Member of the Austrian Academy of Sciences. 